\title{
Quantifying uncertainty on sediment loads using bootstrap confidence intervals
}

\author{
Johanna I. F. Slaets ${ }^{1}$, Hans-Peter Piepho ${ }^{2}$, Petra Schmitter ${ }^{3}$, Thomas Hilger ${ }^{1}$, and Georg Cadisch ${ }^{1}$ \\ ${ }^{1}$ Institute of Plant Production and Agroecology in the Tropics and Subtropics, University of Hohenheim, \\ Garbenstrasse 13, 70599 Stuttgart, Germany \\ ${ }^{2}$ Biostatistics Unit, Institute of Crop Science, University of Hohenheim, Fruwirthstrasse 23, \\ 70599 Stuttgart, Germany \\ ${ }^{3}$ The International Water Management Institute, Nile Basin and East Africa Office, \\ Addis Ababa, Ethiopia
}

Correspondence to: Johanna I. F. Slaets (hanna.slaets@gmail.com)

Received: 27 May 2016 - Published in Hydrol. Earth Syst. Sci. Discuss.: 21 June 2016

Revised: 6 December 2016 - Accepted: 23 December 2016 - Published: 27 January 2017

\begin{abstract}
Load estimates are more informative than constituent concentrations alone, as they allow quantification of on- and off-site impacts of environmental processes concerning pollutants, nutrients and sediment, such as soil fertility loss, reservoir sedimentation and irrigation channel siltation. While statistical models used to predict constituent concentrations have been developed considerably over the last few years, measures of uncertainty on constituent loads are rarely reported. Loads are the product of two predictions, constituent concentration and discharge, integrated over a time period, which does not make it straightforward to produce a standard error or a confidence interval. In this paper, a linear mixed model is used to estimate sediment concentrations. A bootstrap method is then developed that accounts for the uncertainty in the concentration and discharge predictions, allowing temporal correlation in the constituent data, and can be used when data transformations are required. The method was tested for a small watershed in Northwest Vietnam for the period 2010-2011. The results showed that confidence intervals were asymmetric, with the highest uncertainty in the upper limit, and that a load of $6262 \mathrm{Mg}$ year $^{-1}$ had a $95 \%$ confidence interval of $(4331,12267)$ in 2010 and a load of $5543 \mathrm{Mg}$ an interval of $(3593,8975)$ in 2011. Additionally, the approach demonstrated that direct estimates from the data were biased downwards compared to bootstrap median estimates. These results imply that constituent loads predicted from regression-type water quality models could frequently
\end{abstract}

be underestimating sediment yields and their environmental impact.

\section{Introduction}

The environmental impact of processes such as erosion, sedimentation, eutrophication or degradation of aquatic ecosystems can only be quantified through reliable estimates of sediment, nutrient or pollutant loads (Walling and Webb, 1996). Monitoring constituent concentrations alone does not suffice as these provide information on in-stream quality but offer no means to evaluate outcomes such as reservoir siltation, erosion, soil fertility loss and pollution at the watershed scale both on- and off-site. Despite abundant literature developing appropriate procedures for load estimates, most studies do not report a measure of uncertainty on the load (Kulasova et al., 2012).

In this paper, we will use the example of one of the most commonly measured constituents, suspended sediment, but the methodology developed is applicable to any constituent load. For suspended sediment, the most frequently used method to estimate loads is the so-called rating curve method (Gao, 2008; Horowitz, 2008). In this approach, the suspended sediment concentration (SSC) is predicted by some form of least squares regression with (often log-transformed) discharge as the explanatory variable. This approach introduces two sources of uncertainty into the load equation: the 
uncertainty in the sediment concentration equation (the socalled sediment rating curve), and the uncertainty in the discharge, as discharge is usually not measured directly, but rather is predicted from a stage-discharge rating curve, with water level as the predictor variable. Any measure of uncertainty on the constituent load must take into account the uncertainty in both the constituent concentration and the discharge.

Uncertainty of the sediment concentration prediction has been extensively discussed and, depending on the catchment characteristics, generally good concentration predictions are obtained with errors smaller than $15 \%$ (Horowitz, 2008). In some studies, however, the uncertainty is stated to be considerable. Smith and Croke (2005) for example reported that discharge only explained a quarter of the variability in the concentration data. Walling and Webb (1988) suggested that seasonal differences of the relationship between discharge $(Q)$ and constituent concentration, non-simultaneity of $Q$ and concentration peaks during storms, hysteresis and exhaustion effects are the most important causes of inaccuracy in concentration predictions. Few studies to date have taken this error on the discharge rating curve into account explicitly when calculating uncertainty on load estimates (Vigiak and Bende-Michl, 2013; Rustomji and Wilkinson, 2008). Uncertainties of discharge, however, depend on several factors: the method chosen to estimate the discharge, site conditions and the time interval over which water levels are measured (Harmel et al., 2009; Hamilton and Moore, 2012; McMillan et al., 2012; Tomkins, 2014). In general, discharge is estimated more accurately than constituent concentration. Discharge, however, enters the load equation twice - once as predictor variable for the concentration, and once multiplied with the concentration to get the instantaneous load. Therefore, it deserves further investigation whether or not the error on the discharge estimate can safely be ignored, and in which circumstances. Finally, as discharge is frequently used as a predictor for constituent concentration, the two variables are correlated and their errors cannot be assumed to be independent. As a result, there is no textbook formula to estimate the variance of a constituent load.

Therefore, authors that do report a measure of uncertainty often select a method that is specifically geared towards the application of load estimation at hand and not necessarily applicable to other sites, making it hard to compare results throughout the literature. Harmel et al. (2009), for example, developed a software tool to assess the errors introduced from estimating discharge, sample collection, preservation and storage, and lab analysis. In this tool, each of these sources is considered to be the result of random variability following a normal distribution. The sources of error are assumed to be independent of each other, and it is assumed that the errors follow an additive law, but these assumptions will not apply in all situations.

Moatar and Meybeck (2005) assessed uncertainty on nutrient loads by comparing loads based on a random subsam- ple of measurement times, with a high-resolution load that is considered the "true" load. This approach is suitable for testing different methods and different temporal measurement resolutions of load estimation, but it does not assess the uncertainty of the "true" load, as it is assumed that, with sufficiently high sampling frequency (in their case, daily), the measured load is equivalent to the actual load. More recently, two new candidate approaches have emerged to calculate confidence intervals on loads that have the potential to be generally applicable, regardless of the method used to calculate the load and the distributional assumptions made: bootstrap methods (Mailhot et al., 2008; Rustomji and Wilkinson, 2008; Vigiak and Bende-Michl, 2013) and Bayesian methods that result in credibility intervals (Pagendam et al., 2014; Vigiak and Bende-Michl, 2013).

The bootstrap is a Monte Carlo-type method, where a large number $(B)$ of datasets are simulated - either by sampling with replacement from the original data in the case of the non-parametric bootstrap or by sampling from a fitted distribution in the case of the parametric bootstrap (Efron and Tibshirani, 1993). Bootstrap methods, however, were originally developed for independent, identically distributed random variables. In the context of sediment monitoring the assumption is that the observations used to build the regression model are independent in time. This can be realistic in fixedinterval sampling schemes where the sampling time interval is large, or in the case of discharge where measurements to build the stage-discharge relationship are typically taken far apart in time. For sediment concentration, however, flowproportional sampling is often performed to obtain samples at the highest concentrations. Those observations are usually taken closely together during storms and thus most likely are not independent in time (Slaets et al., 2014). Linear mixed models that model the serial correlation provide an alternative to least squares regression to establish a sediment rating curve for this type of data. Lessels and Bishop (2013) similarly found that the inclusion of a temporal autocorrelation component improved the accuracy and decreased the bias in predictions of total phosphorus and nitrogen river loads. If there is serial correlation in the sediment data, it is necessary to use an adjusted version of the bootstrap that retains the serial correlation in the data intact (Lahiri, 2003). Such methods have already been explored in hydrology in relation to the discharge rating curve: Ebtehaj et al. (2010) and Selle and Hannah (2010) use block bootstrap methods to assess uncertainty in and improve robustness of model parameter estimates for discharge prediction.

To assess uncertainty in constituent loads estimated from continuous concentration and discharge predictions where serial correlation is present, we propose a bootstrap-based method to assess uncertainty in constituent loads that can be used with transformed data, that accounts for the uncertainty in both the sediment rating curve and the stage-discharge rating curve, and that allows for serial correlation in the time series data. We checked whether any of these requirements can 
safely be neglected in certain circumstances, and how they affect the resulting confidence intervals. The corresponding code in SAS was created and is available online to accommodate these different scenarios (https://www.uni-hohenheim. de/bioinformatik/beratung/index.htm).

Our specific aims were: (i) to establish a generally applicable method to calculate confidence intervals on constituent loads, using bootstrap methods, (ii) to account for serial correlation in the data, (iii) to assess whether or not the effect of the uncertainty on discharge is negligible, (iv) to evaluate how data transformations affect the calculations, and (v) to determine the number of bootstrap replicates required to obtain reliable confidence intervals. Combining these aspects, the proposed method provides a means to assess uncertainty on any type of constituent load which was calculated from continuous constituent concentration and discharge predictions estimated with regression-type methods. The approach thus allows load estimates to be reported with an uncertainty assessment, rather than as a point estimate alone, making them informative to end users and decision makers.

\section{Material and methods}

\subsection{Discharge and sediment concentration}

Discharge and suspended sediment concentrations were continuously monitored for a period of 2 years (1 January 2010 31 December 2011) in a small agricultural catchment in mountainous Northwest Vietnam. The catchment is located in the Chieng Khoi commune $\left(21^{\circ} 7^{\prime} 60^{\prime \prime} \mathrm{N}, 105^{\circ} 40^{\prime} 0^{\prime \prime} \mathrm{E}\right.$, $350 \mathrm{~m}$ a.s.l), Yen Chau district, in the tropical monsoon belt where the rainy season begins in April and ends in October. Average annual precipitation is around $1200 \mathrm{~mm}$; average annual temperature is $21^{\circ} \mathrm{C}$. The occurrence of typhoons is not uncommon especially at the end of the rainy season, and daily rainfall amounts can rise to $200 \mathrm{~mm}$. The largest storm during the 2 years of this study was on 12 July 2011 and consisted of $73 \mathrm{~mm}$ of rainfall in $3 \mathrm{~h}$. The dominant soils are Alisols and Luvisols (Clemens et al., 2010). The landscape has an altitudinal range between 320 and $1600 \mathrm{~m}$ a.s.l. with slopes ranging from 0.05 to $65 \%$. The measurement location is in a river at the outlet of a small watershed with a contributing area of $2 \mathrm{~km}^{2}$, of which $0.6 \mathrm{~km}^{2}$ consist of paddy fields. A 26.3 ha surface reservoir with a buffering capacity of $10^{6} \mathrm{~m}^{3}$ provides irrigation water for rice production via concrete irrigation channels, and the paddies drain into the monitored river. As the reservoir fills up with the progression of the rainy season, excess water is removed via a spillover which drains into the river, typically from July till October. During this period, discharge in the river is an order of magnitude larger than during times when the spillover is not active: mean daily discharge in the dry season equaled $0.08 \mathrm{~m}^{3} \mathrm{~s}^{-1}$ (with a standard deviation of $0.13 \mathrm{~m}^{3} \mathrm{~s}^{-1}$ ), while mean daily discharge with the spillover active amounted to $1.22 \mathrm{~m}^{3} \mathrm{~s}^{-1}$ (with a standard deviation of $0.66 \mathrm{~m}^{3} \mathrm{~s}^{-1}$ ).

For the discharge monitoring, water levels were measured every $2 \mathrm{~min}$ for the river station using pressure sensors (EcoTech, Germany). The stage-discharge relationship was established with the velocity-area method (Herschy, 1995), where the velocity is measured with a propeller-type current meter (OTT, Germany) at one or more points in each vertical, depending on the water depth. The discharge is subsequently derived from the sum of the product of mean velocity, depth and width between verticals. Discharge measurements were never taken on the same day, and the closest time interval between two measurements was 1 week. The estimated discharge $\hat{Q}$ in $\mathrm{m}^{3} \mathrm{~s}^{-1}$ at time $i$ was then predicted from

$\log \hat{Q}_{i}=\log \hat{\alpha}+\hat{\gamma} \log \left(h_{i}-\beta\right)$,

where $h_{i}$ is the water level (in m) at time $i, \hat{\alpha}$ and $\hat{\gamma}$ the estimated rating curve constants and $\beta$ the measured sensor offset, with $\hat{\alpha}$ and $\hat{\gamma}$ estimated using the method of least squares on the log-transformed scale. This transformation was done to stabilize the variance.

As the irrigation management disturbed the natural relationship between $Q$ and SSC, a turbidity-based method was used to monitor SSC. Turbidity was measured every $2 \mathrm{~min}$ with NEP395 sensors (McVan, Australia); 228 water samples were collected using a storm-based approach, by taking around 20 grab samples per sampled rainfall event in order to accomplish the best possible coverage of all concentration ranges; 188 storm-flow samples were collected during 24 rainfall events over the 2-year duration of the study. Additionally, every 2 weeks a base-flow sample was taken. The sediment concentration was determined gravimetrically on a sample of $500 \mathrm{~mL}$ by letting it settle overnight in refrigerated conditions, prior to siphoning off the supernatant and drying the remaining sediment at $35^{\circ} \mathrm{C}$, as is recommended for samples with very high sediment concentrations (ASTM, 2013).

Rainfall was quantified with a tipping-bucket rain gauge on a weather station (Campbell Scientific, USA). Events were defined based on rainfall data (no pause in precipitation for longer than $30 \mathrm{~min}$ ) and lag times were added based on cross-correlation analysis as described in Schmitter et al. (2012). A total of 420 rainfall events took place and were monitored during the 2-year study period.

Continuous sediment concentrations were then obtained from a mixed model described in Slaets et al. (2014). The response variable, sediment concentration, was Box-Coxtransformed to stabilize the variance using the SAS macro described in Piepho (2009). The optimal value of the transformation parameter was estimated by the maximum likelihood method, and the selected value was the log transformation. Other transformations, such as the square root, were inspected using residual plots and were found to be unsuitable for meeting the assumptions of normality and homoscedasticity. Predictor variables were chosen with forward selection 
based on the Akaike information criterion (AIC). The model uses turbidity and discharge as quantitative predictor variables, and accounts for serial correlation. As surface reservoir irrigation management was present in the watershed, classic variables related to catchment characteristics such as hysteresis patterns and exhaustion effects were not suitable predictors of sediment concentration. The predictor variables turbidity and discharge were also log transformed. All samples from the 2-year study period were used to build the concentration prediction model, and load estimates from both years are thus predicted from the same model with the same parameter estimates. All statistical analyses were performed using the MIXED procedure of SAS 9.4, which can fit linear models with more than one random effect. The covariance structure used to model serial correlation in the present study was a first-order autoregressive (AR(1)) model, which was selected based on the AIC. Assumptions of normality and homogeneity of variance were checked visually using diagnostic plots.

Conceptually, the concentration prediction error can also be separated into an underlying latent autoregressive process generating the true concentrations, and an independently distributed measurement error corresponding to white noise in time series data. The white noise is equal to the error that would remain if two measurements were conducted at almost coinciding time points. This variability is typically attributable to measurement error and in spatial statistics, this is what is known as a nugget effect. In the MIXED procedure, this effect was fitted by using the local option in the repeated statement.

Validation was performed using 5-fold cross validation, in which the dataset is split randomly into five parts, and each part is used four times to calibrate the model, and one time for validation, so that each observation in the dataset is used for validation once. Pearson's correlation coefficient $(r)$ was calculated between the observed and predicted values resulting from the validation. A SAS macro that performs $k$-fold cross validation ( $k=5$ in our case) for linear mixed models using the MIXED procedure is described in Slaets et al. (2014). Additionally, event-based 5-fold cross validation was performed, where all samples belonging to single events were resampled jointly, rather than individual observations.

\subsection{Bootstrap resampling procedure}

In the non-parametric bootstrap, a large number $(B)$ of random samples, of the same size as the original dataset, is drawn by sampling observations with replacement from that original dataset. For each of the $B$ bootstrap samples, the sample statistic of interest (in this case, the sediment load) is calculated and from the resulting empirical distribution, measures of uncertainty can be obtained. As this empirical distribution is only a good approximation of the true distribution when the bootstrap resampling mechanism is able to recreate the original sampling process (Efron and Tibshirani,
1993), we need to understand the sampling processes resulting in the annual sediment load. Since neither discharge nor constituent concentration are measured continuously, annual loads are normally estimated by calculating the sum of instantaneous loads, measured at equally spaced discrete points in time. The load at a time $i$ is then generated from

$\hat{L}_{i}=\hat{Q}_{i} \times \hat{C}_{i}$,

where $\hat{L}_{i}$ is the estimated instantaneous load at time $i$ in $\mathrm{g} \mathrm{s}^{-1}, \hat{Q}_{i}$ is the estimated discharge at time $i$ in $\mathrm{m}^{3} \mathrm{~s}^{-1}$ and $\hat{C}_{i}$ is the estimated concentration at time $i$ in $\mathrm{g} \mathrm{m}^{-3}$. These instantaneous loads are multiplied by a time factor accounting for the monitoring interval. In the present study, for example, the factor was $120 \mathrm{~s}$, as measurements were done every 2 min. Monthly or annual loads in $\mathrm{Mg}$ can then be calculated by simply summing up the instantaneous loads for the whole time interval and multiplying by a factor $10^{-6}$ to convert from $\mathrm{mg}$ to $\mathrm{Mg}$ :

$\hat{L}_{1 \text { to } t}=\sum_{i=1}^{t}\left(\hat{L}_{i} \times 120 \times 10^{-6}\right)$.

Looking at Eq. (2) for the load estimate at a time $i$, there are really two separate sampling processes from two distinct populations at work in the load estimation: firstly the sampling for the discharge rating curve (pairs of $Q$ and $h$ from the full time series of $Q$ and $h$ pairs), and secondly, the samples used to build the sediment rating curve (observations of $C$ and hydrological predictor variables from the full time series of $C, Q$ and turbidity). In order to assess the uncertainty of the discharge equation, the bootstrap replicates can be created by simply sampling $(Q, h)$ pairs at random with replacement from the original dataset. Simple random resampling assumes independence, which is dependent on the monitoring scheme: in the case of our dataset, discharge measurements were never taken on the same day, and the smallest interval between two measurements was 1 week. In order to test this assumption, an $\mathrm{AR}(1)$ variance-covariance structure was fitted to the discharge data. As the AIC showed an increase of two points compared to an independent structure, no serial correlation was present in the $(Q, h)$ pairs. Using Eq. (1), bootstrap discharges $\hat{Q}_{i}^{*}$ can be generated according to

$\log \hat{Q}_{i}^{*}=\log \hat{\alpha}^{*}+\hat{\gamma}^{*} \log \left(h_{i}-\beta\right)$,

where $h_{i}$ is the water level at time $i, \beta$ is the measured sensor offset, and $\hat{\alpha}^{*}$ and $\hat{\gamma}^{*}$ are the bootstrap estimates of the discharge rating curve parameters. These $B$ predictions of $Q, B$ being the number of bootstrap repetitions, must subsequently be fed into the bootstrapped sediment rating curve, as discharge typically is one of the predictor variables for SSC. But the previously described resampling mechanism cannot be applied to the observations used to build the sediment rating curve, as the simple random sampling assumes that the observations are independent. Water samples are 
often collected in a storm-based approach, as was done in this study, where they were collected sometimes only minutes apart during rainfall events. For these types of hydrological datasets where temporal autocorrelation is present, Ebtehaj et al. (2010) recommended the use of specialized sampling procedures that keep the serial correlation intact, such as the moving block bootstrap, the circular block bootstrap or the stationary block bootstrap, described in detail by Lahiri (2003) and applied to constituent concentrations by Hirsch et al. (2015).

Among these specialized methods, no preferred method has emerged from the literature. Furthermore, many of these methods require a vast set of decisions such as for example the block size for which no general recommendation exists. As a consequence, results from different methods are not straightforward to compare. As the goal of the bootstrap is to mimic the original sampling process, however, there is an intuitive choice in the case of event-based sampling: the rainfall events form natural "blocks" or sampling units, which is why water quality models used to predict continuous time series and thus new events should be validated on an event basis, rather than on a sample basis (Lessels and Bishop, 2013). So rather than sampling with replacement from the individual observations (water samples representing a single time point), all samples belonging to one event can be resampled with replacement, thus keeping all observations within one event together and maintaining the serial correlation intact.

On the other hand, base-flow samples are typically taken at fixed time intervals far apart in time (here every 2 weeks). They can therefore be considered to be independent and can be resampled by simple random sampling with replacement, thus bootstrapping individual water samples from single time points. In a previous model published in Slaets et al. (2014), we explored the use of several alternative variance-covariance structures to model the serial correlation. The selected spatial power model unfortunately caused non-convergence for a large number of the bootstrap replicates when using it for bootstrap load estimates, and therefore the AR(1) structure was implemented as it did not have convergence issues. The difference in AIC between the AR(1) and spatial power models was four points. Therefore the spatial power model is most likely the best performing model, but there is still considerable support for the AR(1) model (Burnham and Anderson, 2002). The spatial power structure with time as the coordinate showed that the autocorrelation becomes nearly zero for samples taken more than $80 \mathrm{~min}$ apart - which coincides with the average duration of rainfall events. Therefore the base-flow samples were considered to be independent. An increase in AIC of one point when fitting a first-order autoregressive covariance structure confirmed the lack of serial correlation in the base-flow samples. By resampling events with replacement for the storm-flow samples and observations with replacement for the base-flow samples, $B$ time series of predicted sediment concentration
$\hat{C}_{i}^{*}$ are generated from

$\hat{C}_{i}^{*}=\hat{\delta}^{*}+\left(\hat{v}^{*}\right)^{T} X_{i}$,

with $\hat{\delta}^{*}$ and $\hat{v}^{*}$ the bootstrap estimates of the intercept and the regression coefficients, respectively, $\left(\hat{v}^{*}\right)^{T}$ the transpose of vector $\hat{v}^{*}$ and $X_{i}$ the design vector of the fixed effects. This is a generalized equation applicable to any linear model, regardless of the number of predictor variables. If the sediment concentration was predicted using turbidity and discharge, for example, the bootstrap time series of predicted sediment concentration would be generated from

$\hat{C}_{i}^{*}=\hat{\delta}^{*}+\hat{\eta}^{*} \hat{Q}_{i}^{*}+\hat{\kappa}^{*} T_{i}$,

where $\hat{\eta}^{*}$ is the bootstrap parameter estimate of the regression coefficient for $\hat{Q}_{i}^{*}$, the bootstrap discharge at time $i$ generated from Eq. (4), and $\hat{\kappa}^{*}$ the bootstrap parameter estimate of the regression coefficient for $T_{i}$, the turbidity at time $i$, respectively.

This resampling process accounts for the uncertainty that arises from estimating the parameters of the sediment rating curve from a dataset with a limited number of observations. If there were an unlimited number of water samples available, the uncertainty of these parameter estimates would decrease to zero. But it is more realistic to assume that, even if there were a very large number of samples available, there would still remain scatter in the real constituent concentrations around the equation, as the equation simply does not fully explain all the variation in sediment concentration. Sediment loads vary not only with discharge, but also with upstream sediment supply, which in turn depends additionally on geology, soil types, land cover and land use change or management, all influencing sediment quantity and quality (Walling, 1977). Therefore, there is a fundamental reason for the scatter in the data: sediment loads are inherently noncapacity loads. Even if there were an unlimited number of samples available, this would not result in a perfect equation to predict sediment concentration. Therefore this additional uncertainty needs to be taken into account. For the discharge rating curve, if the river bed is stable and the stream bank vegetation does not change, the stage-discharge equation has a high accuracy and it is reasonable to assume the only error in the equation is measurement error; therefore, this additional uncertainty is not a concern.

To introduce this second source of error on the sediment rating curve, Rustomji and Wilkinson (2008) and Vigiak and Bende-Michl (2013) added an additional step to the bootstrap process: a randomly drawn residual from the original regression equation was added to the expected value of the constituent concentration, so that the predicted concentration included both the uncertainty of the parameters of the rating curve due to having a finite sample, and the uncertainty that arises from the fact that sediment concentrations simply cannot be perfectly predicted by any equation, regardless of 

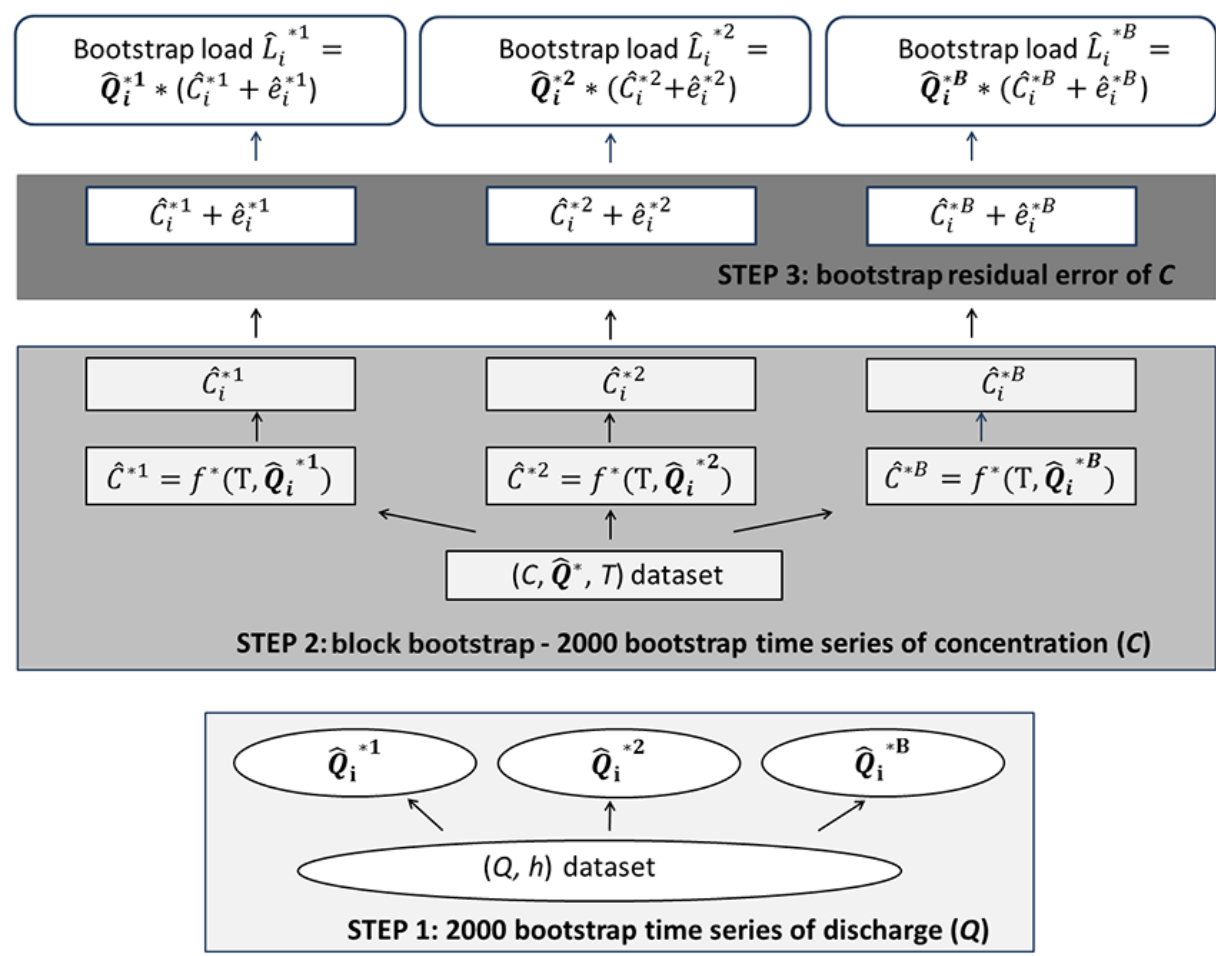

Figure 1. Flowchart showing the three-step bootstrap mechanism.

how large the observed dataset would be. However, by randomly resampling from the residuals, it is assumed that these residuals are independent.

When this assumption does not hold because samples are taken very closely together in time, as was the case for our dataset, the method can be modified so that the added errors reflect the temporal autocorrelation. To this end, the covariance parameter estimates from the original sample can be used as plug-in estimates. In the present dataset, an $\operatorname{AR}(1)$ structure was fitted to the data (Verbeke and Molenberghs, 2009), resulting in two covariance parameter estimates: one for the autocorrelation parameter $(\hat{\rho})$ and one for the residual error variance $\left(\hat{\sigma}_{e}^{2}\right)$. The restricted maximum likelihood algorithm was used to simultaneously estimate the fixed effects and the covariance structure (Patterson and Thompson, 1971). The use of the covariance parameter estimates obtained assuming a normal distribution of errors implies that the method is partly parametric. This is necessary in order to take the serial correlation in the data into account. The bootstrap error term $e^{*}$ at time $i$ was then generated according to the following equation:

$\hat{e}_{i}^{*}=\hat{\rho}^{*} \hat{e}_{i-1}^{*}+\sqrt{\left(1-\left(\hat{\rho}^{*}\right)^{2}\right)} \times f_{i}^{*}$,

where $\hat{\rho}^{*}$ is the bootstrap estimate of the autocorrelation parameter, and the error $f_{i}^{*}$ was randomly drawn from a normal distribution with mean zero and a bootstrap variance $\left(\hat{\sigma}_{e}^{*}\right)^{2}$. The bootstrap prediction of a sediment concentration at time $i$, including the error expected due to residual scatter in the data, is then given by

$\hat{C}_{i}^{*}+\hat{e}_{i}^{*}=\hat{\delta}^{*}+\left(\hat{v}^{*}\right)^{T} X_{i}+\hat{\rho}^{*} \hat{e}_{i-1}^{*}+\sqrt{\left(1-\left(\hat{\rho}^{*}\right)^{2}\right)} \times f_{i}^{*}$.

In summary, the complete bootstrap process that accounts for uncertainty in the parameter estimates of both the discharge and sediment rating curves and uncertainty due to residual scatter in the sediment concentrations consists of three steps (Fig. 1):

1. resampling with replacement from the $(Q, h)$ pairs $B$ times, in order to get $B$ bootstrap stage-discharge equations; applying these equations to the continuous water level data to obtain $B$ bootstrap time series $\left(Q^{*}\right)$ for discharge;

2. block-bootstrapping the $\left(C\right.$, turbidity, $Q^{*}$, rainfall $)$ dataset by drawing whole events and base-flow samples with replacement, in each replicate plugging in the corresponding bootstrap $Q^{*}$ from Step 1, in order to get $B$ bootstrap sediment rating curves; then applying these bootstrap sediment rating curves to the continuous turbidity, $Q^{*}$, and rainfall data to obtain $B$ time series for the continuous suspended sediment concentration; and

3. adding an error term to the concentration predictions to account for the residual scatter that is inherent to the sediment concentration. 
In order to obtain a bootstrap estimate of the instantaneous load $L$ at time $i$, the equation is

$\hat{L}_{i}^{*}=\hat{Q}_{i}^{*} \times\left(\hat{C}_{i}^{*}+\hat{e}_{i}^{*}\right)$

The residual scatter on the discharge is not added, as the stage-discharge rating curve has a much higher accuracy on the one hand, and on the other hand, velocity measurements are typically taken quite far apart in time, which would not allow modeling of the serial correlation of the time series for discharge, but this would be needed because of the shortness of the time intervals considered here ( $2 \mathrm{~min}$ ).

Finally, these bootstrap instantaneous load estimates can be summed up for the whole time interval, resulting in $B$ estimates of monthly or annual loads:

$\hat{L}_{1 \text { to } t}^{*}=\sum_{i=1}^{t}\left(\hat{L}_{i}^{*} \times 120 \times 10^{-6}\right)$.

\subsection{Data transformations}

If the data are not normally distributed, it can be necessary to transform variables, as was done for this dataset with a BoxCox transformation. In this case, the variables in question can simply be transformed before starting the bootstrap, and all the bootstrap estimates are obtained on the transformed scale. The back-transformation is then performed in Eq. (9) to obtain load estimates on the original scale. For example, in a typical case where both discharge and sediment concentration need to be log-transformed, the bootstrap predictions of discharge in Eq. (4) and of concentration in Eq. (8) will be on the log scale. These predictors then need to be backtransformed to the original scale using the inverse of the logarithm.

This approach is applicable to any type of data transformation, and thus offers a flexible framework that can accommodate different methods of estimating the constituent concentration. However, if a modeled residual error term $\hat{e}_{i}^{*}$ is not included, care must be taken with the back-transformation. With nonlinear data transformations (the log-transformation and the Box-Cox transformation being prime examples), predicted means cannot be naïvely back-transformed and interpreted as means on the original scale. Correction factors can be applied that compensate for the underestimation of SSC that arises from doing the predictions on the transformed scale. A commonly used non-parametric correction factor is Duan's smearing estimator (Duan, 1983), where the sample average of the exponentiated residuals from the model is used as the correction factor. Duan's smearing estimator assumes independent and identically distributed errors, however, and is therefore also not a suitable alternative when serial correlation in the data is present. Alternatively, as pointed out by Rustomji and Wilkinson (2008), adding the modeled residual error removes the need to apply a correction factor and is therefore the recommended approach. Regardless of the chosen correction factor, it is important that homoscedasticity after the transformation is confirmed by visually inspecting the diagnostic plots, as was done in the case of this dataset. While discharge is also typically predicted on the log-transformed scale, in our dataset the variance was much smaller than that of the concentration data. With a small variance, the log-normal distribution is nearly normal and, therefore, the naïve back-transformation of $\log \left(\hat{Q}_{i}\right)$ should approximate the mean well.

\subsection{Alternative option to simulate errors}

If a data transformation is required and one does not want to explicitly simulate the residual scatter, then a correction factor must be applied to the back-transformed concentration. This correction is needed because the naïve backtransformation (for example, taking the exponent of the predictions if the predictions are on the log scale) does not yield a predicted mean, but rather a predicted median. While medians can be informative measures of a central tendency to skewed datasets, they are not appropriate when the objective is to calculate a constituent load: loads are sums over equally spaced time points, and in order to obtain an unbiased estimate of this sum over time intervals, we need to sum up estimates of the expected values, rather than the medians, for each interval.

The required correction factor is specific to the type of data transformation. For a logarithmic transformation, the expected value can be obtained by adding on half of the residual error variance to the predicted concentration on the log scale before back-transforming. For other cases of the Box-Cox transformation, the correction depends on the selected transformation parameter. Solutions for specific examples of the transformation parameter can be found in Freeman and Modarres (2006). As the selected transformation in this dataset was the logarithm, the correction of adding half the residual error variance before back-transforming was compared to the approach where the error is simulated, in order to see how this affects sediment load estimates and the resulting confidence intervals.

\subsection{Bootstrap confidence intervals}

A straightforward way to calculate a confidence interval (CI) on a parameter after bootstrapping is the bootstrap percentile method (Efron and Tibshirani, 1993). If a $95 \%$ CI is required, the confidence interval would simply be calculated by ordering the bootstrap load estimates from small to large and taking the 2.5th and 97.5th percentiles as the lower and upper limits.

This method was used by Rustomji and Wilkinson (2008) on sediment loads and is transformation-respecting, also when the sample statistic is not normally distributed (Efron and Tibshirani, 1993). This property is important in the case of loads, because data are typically log-transformed. As a confidence interval depends on the tail of the empirical bootstrap distribution where fewer samples occur, a relatively 
large number of bootstrap replicates (upward of 500) are usually required to achieve acceptable accuracy (Efron and Tibshirani, 1993). How many exactly depends on the statistic in question, and should be empirically tested for each case: when the process is repeated, the resulting CI should not greatly differ, otherwise the number is too small. In the present dataset, a choice of 2000 bootstrap replicates yielded replicable results.

Improving upon the bootstrap percentile method, Efron and Tibshirani (1993) proposed bias-corrected and accelerated intervals, used by Vigiak and Bende-Michl (2013). Unfortunately, this approach requires an even larger number of bootstrap replicates than the percentile method to sufficiently reduce the Monte Carlo sampling error. This is a disadvantage when working with hydrological time series, as the datasets typically contain a large number of records already. This method then quickly becomes time consuming, and therefore in this paper, preference was given to the more intuitive and less computationally intensive bootstrap percentile method.

\subsection{Identifying hydrological drivers of uncertainty}

The proposed three-step bootstrap process offers an opportunity to assess the importance of different aspects of the load calculation for the accuracy of the estimate. By leaving out step 1 (bootstrapping the $Q-h$ pairs) and just using $Q$ as predicted by the discharge rating curve from all observed data points, confidence intervals can be obtained that only take into account the uncertainty on the sediment rating curve. If the resulting confidence intervals closely resemble the confidence intervals calculated with the full approach, this would mean that the uncertainty in the sediment concentration is what drives the uncertainty in the loads, thus supporting the finding that the error in the discharge is negligible compared with other sources of uncertainty (e.g., Némery et al., 2013, Vigiak and Bende-Michl, 2013).

As the accuracy of the stage-discharge relationship depends on the type of streambed, the method chosen and the number of measurements taken, this assumption might also hold true for some watersheds such as the one in this study, where the relationship had a high $R^{2}$, but not for others. To determine at which point the uncertainty in $Q$ must be taken into account for the load confidence interval, datasets of $(Q$, h) pairs were simulated with decreasing $R^{2}(0.95,0.90,0.85$ and 0.80 ), and were each used as an input dataset for bootstrapping the stage-discharge relationship (Step 1 in Fig. 1) in order to test the sensitivity of the confidence intervals to the accuracy of the discharge rating curve. The datasets with a fixed realized $R^{2}$ were simulated by a rescaling of errors which is described in Appendix A, and SAS code to perform the simulation can be found in the Supplement.

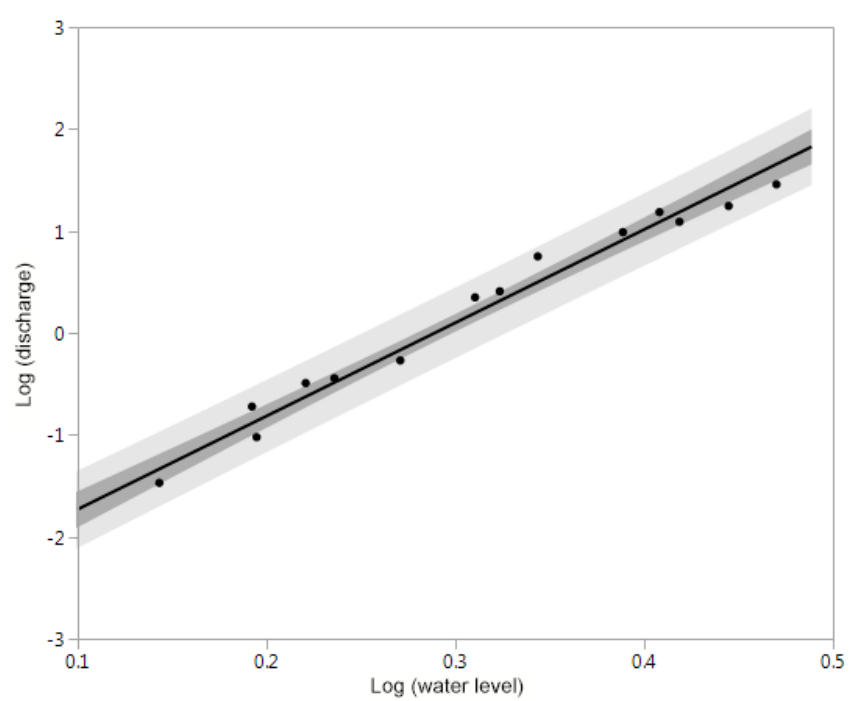

Figure 2. Discharge rating curve plotted on the log-transformed scale showing the $95 \%$ confidence interval for the regression line (dark grey) and for new predictions (light grey). Stage-discharge rating curve: $\log ($ discharge $)=(9.0819 \cdot \log ($ water level $))-2.6423$ $\left(n=15, R^{2}=0.98\right)$.

\section{Results}

\subsection{Rating curves and load estimates}

The coefficient of determination of the stage-discharge relationship was 0.98 ( $n=15$, Fig. 2). Homoscedasticity was observed on the log-transformed scale (Fig. 3). For the sediment rating curve, Pearson's $r$ between observed and predicted values on the log-transformed scale was $r=0.75$ after 5 -fold cross validation ( $n=228$, Fig. 4). Event-based cross validation yielded very similar results, demonstrating the robustness of the model $(r=0.77)$. The sediment rating curve tends to overpredict low concentrations and underpredict high concentrations for new data, as is visible in Fig. 4. This tendency of regression towards the mean is typically seen when models are fitted to very noisy data, and is also well documented in erosion studies (Nearing, 1998). Thus, in the case of our dataset, the discharge rating curve explained a higher proportion of the variance than the sediment rating curve, as is typical. Again, homoscedasticity was observed on the logtransformed scale (Fig. 5). The bootstrap parameter estimates for $\rho$ of the AR(1) process varied from 0.56 to 0.93 with a mean of 0.77, showing the block bootstrap kept the serial correlation intact as required.

The size of the estimated load depended on the method chosen for estimation. First, the load was calculated directly from the model estimates based on the full datasets, without bootstrapping (Direct estimate in Table 1). The sediment concentrations in this case were back-transformed by applying the correction appropriate for log-transformed data, which is to add half the residual error variance before back- 
Table 1. Annual sediment load estimates (in Mg per year) for the 2 years of the study directly estimated without bootstrapping, and load estimates with $95 \%$ confidence interval limits and interval widths (difference between the upper and lower limits) for the three different bootstrap methods: the full method shown in Fig. 1, the method without modeled error (i.e., leaving out Step 3 in Fig. 1) and the method without bootstrapping discharge (i.e., leaving out Step 1 in Fig. 1) (n/a: not applicable).

\begin{tabular}{|c|c|c|c|c|c|c|c|c|c|c|}
\hline \multirow[b]{2}{*}{ Method } & \multicolumn{2}{|c|}{ Error source } & \multicolumn{4}{|c|}{2010} & \multicolumn{4}{|c|}{2011} \\
\hline & Autocorrelation & $Q$-equation & Estimate & Lower & Upper & Width & Estimate & Lower & Upper & Width \\
\hline & & & \multicolumn{4}{|c|}{$\mathrm{Mg} \mathrm{a}^{-1}$} & \multicolumn{4}{|c|}{$\mathrm{Mg} \mathrm{a}^{-1}$} \\
\hline Direct estimate & & & 5607 & $\mathrm{n} / \mathrm{a}$ & $\mathrm{n} / \mathrm{a}$ & $\mathrm{n} / \mathrm{a}$ & 4997 & $\mathrm{n} / \mathrm{a}$ & $\mathrm{n} / \mathrm{a}$ & $\mathrm{n} / \mathrm{a}$ \\
\hline Full bootstrap method & $\checkmark$ & $\checkmark$ & 6262 & 4331 & 12267 & 7936 & 5543 & 3593 & 8975 & 5383 \\
\hline Bootstrap without modeled error & & $\checkmark$ & 6575 & 4372 & 14586 & 10214 & 5839 & 3713 & 10410 & 6697 \\
\hline Bootstrap without discharge & $\checkmark$ & & 5944 & 4203 & 11649 & 7446 & 5413 & 3521 & 8394 & 4876 \\
\hline
\end{tabular}

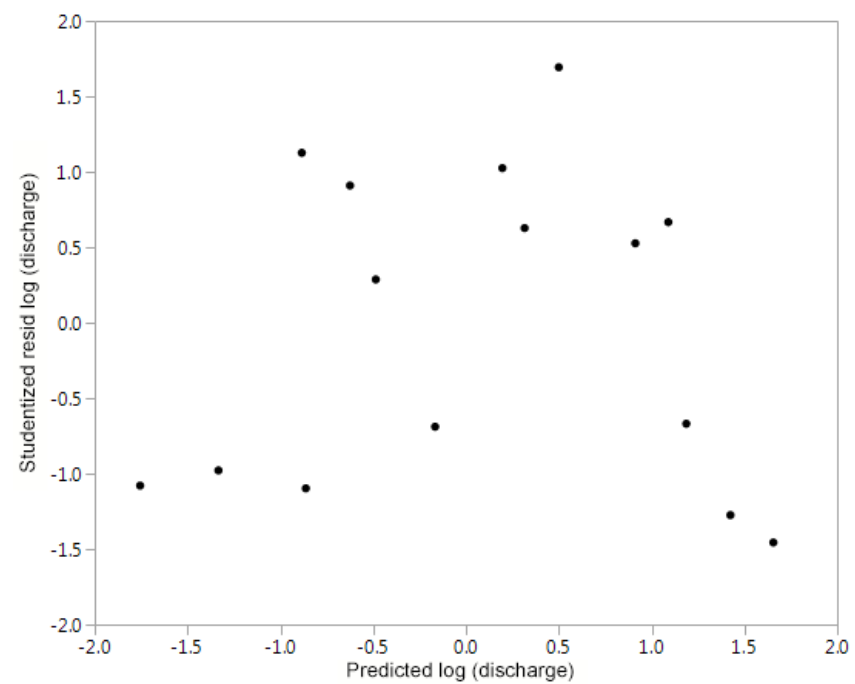

Figure 3. Residual plot for the discharge rating curve, showing studentized residuals versus the predicted discharge (on the logtransformed scale).

transformation. Second, the median of the bootstrap estimates of the sediment load was taken, where, identically to the first case, the concentrations were corrected by adding half the residual error variance before back-transforming (Bootstrap without modeled error in Table 1). Third, the median of the bootstrap estimates was taken for the bootstrap process that included a modeled, autoregressive error term (Full bootstrap method in Table 1).

For this last estimation method, the annual sediment load was estimated to be $6262 \mathrm{Mg}$ in 2010 and $5543 \mathrm{Mg}$ in 2011 (Table 1). When the median from the bootstrap sediment load estimates was taken without modeled error, but rather applying the back-transformation correction, the load was approximately $5 \%$ higher for both annual and monthly load estimates (Table 1 and Fig. 6). The annual loads thus amounted to $6575 \mathrm{Mg}$ in 2010 and $5839 \mathrm{Mg}$ in 2011. Finally, if sediment loads were estimated not by bootstrapping, but directly from the data, then the results were around $10 \%$ lower com-

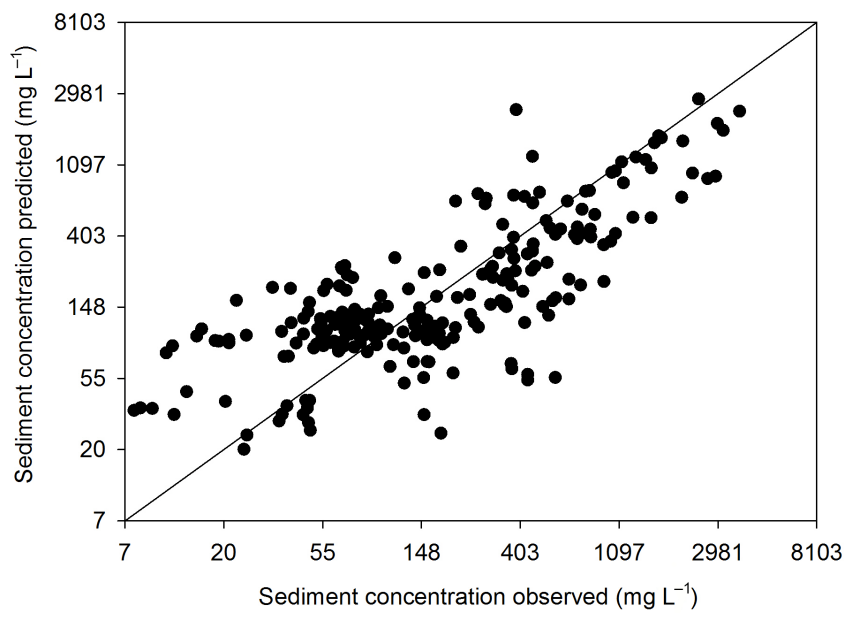

Figure 4. Observed versus predicted values of the sediment rating curve. Predictions are from the linear mixed model with turbidity and discharge as quantitative predictor variables, and after 5 -fold cross validation $\left(n=228, r^{2}=0.56\right)$. Axes are on the $\log$ transformed scale, while tick labels show values on the original scale.

pared to the first estimates, at 5607 and $4997 \mathrm{Mg}$, respectively, in 2010 and 2011.

In all three approaches the difference between the 2 years remained consistent and all estimates were within the bounds of the confidence intervals, both for those calculated by modeling error and those calculated by adding half the variance before back-transformation.

In this particular $2 \mathrm{~km}^{2}$ catchment, the annual sediment export of 6262 and $5543 \mathrm{Mg}$ cannot be interpreted as resulting in average erosion rates of approximately $30 \mathrm{Mg} \mathrm{ha}^{-1}$ due to the irrigation management in the catchment. A large part of the sediments are not eroded within the watershed but released from the irrigation reservoir, which has a contributing area of 490 ha, either via the irrigation channels or through a spillover mechanism which releases excess water when the reservoirs maximum capacity is reached. In 2011, the former mechanism introduced around $800 \mathrm{Mg}$ of sediments to the catchment, and the latter resulted in a load of $1556 \mathrm{Mg}$ 


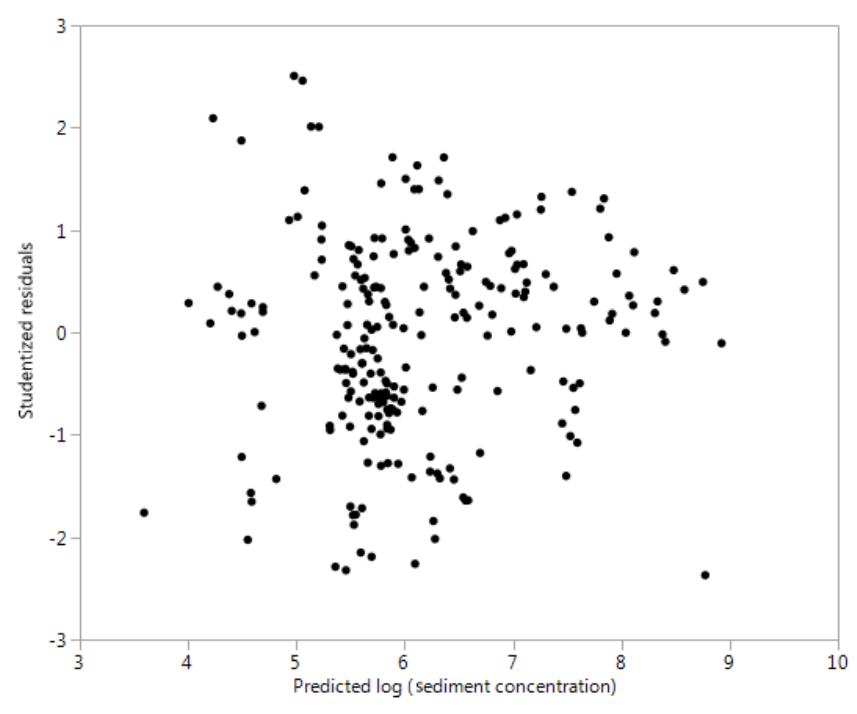

Figure 5. Residual plot of the sediment concentration prediction model; studentized residuals versus the predicted sediment concentration (on the log-transformed scale).

(Slaets et al., 2015). True upland area erosion rates were estimated at $7.5 \mathrm{Mg} \mathrm{ha}^{-1} \mathrm{a}^{-1}$ (Slaets et al., 2015).

\subsection{Width of confidence intervals for sediment loads}

Before looking at the bootstrap confidence intervals, the histograms of the bootstrap load estimates were evaluated (Fig. 7). The histogram of the 2000 bootstrap estimates looked reasonably smooth, so we concluded that sample size was adequate for the percentile bootstrap. When reducing the number of bootstrap replicates (Fig. 8), the change in smoothness, especially in the right tail, becomes visible. Tail smoothness of the empirical distribution is a requirement when using the percentile method to obtain confidence intervals (Efron and Tibshirani, 1993). At 500 bootstrap replicates, the center of the distribution displays a lack of smoothness as well, thus not only affecting the confidence interval estimates, but the load estimates as well. For both years and both for the full method and the method without modeled error, the histograms were found to be skewed to the right, even when the loads were log-transformed. This skewedness means that, in the case of our dataset, the assumption of normality would not hold for estimated annual loads.

As a result of the distribution of the loads, the confidence intervals were always asymmetric, with the difference between the upper limit and the estimate around $80 \%$ larger than the difference between the estimate and the lower limit. The width of the intervals - the difference between the upper and lower limits of the interval - varied between years and between methods, while remaining on the same order of magnitude (Table 1). In 2010, the interval was always wider, regardless of which method was chosen, for the annual as well as the monthly loads (Table 1 and Fig. 6). The year
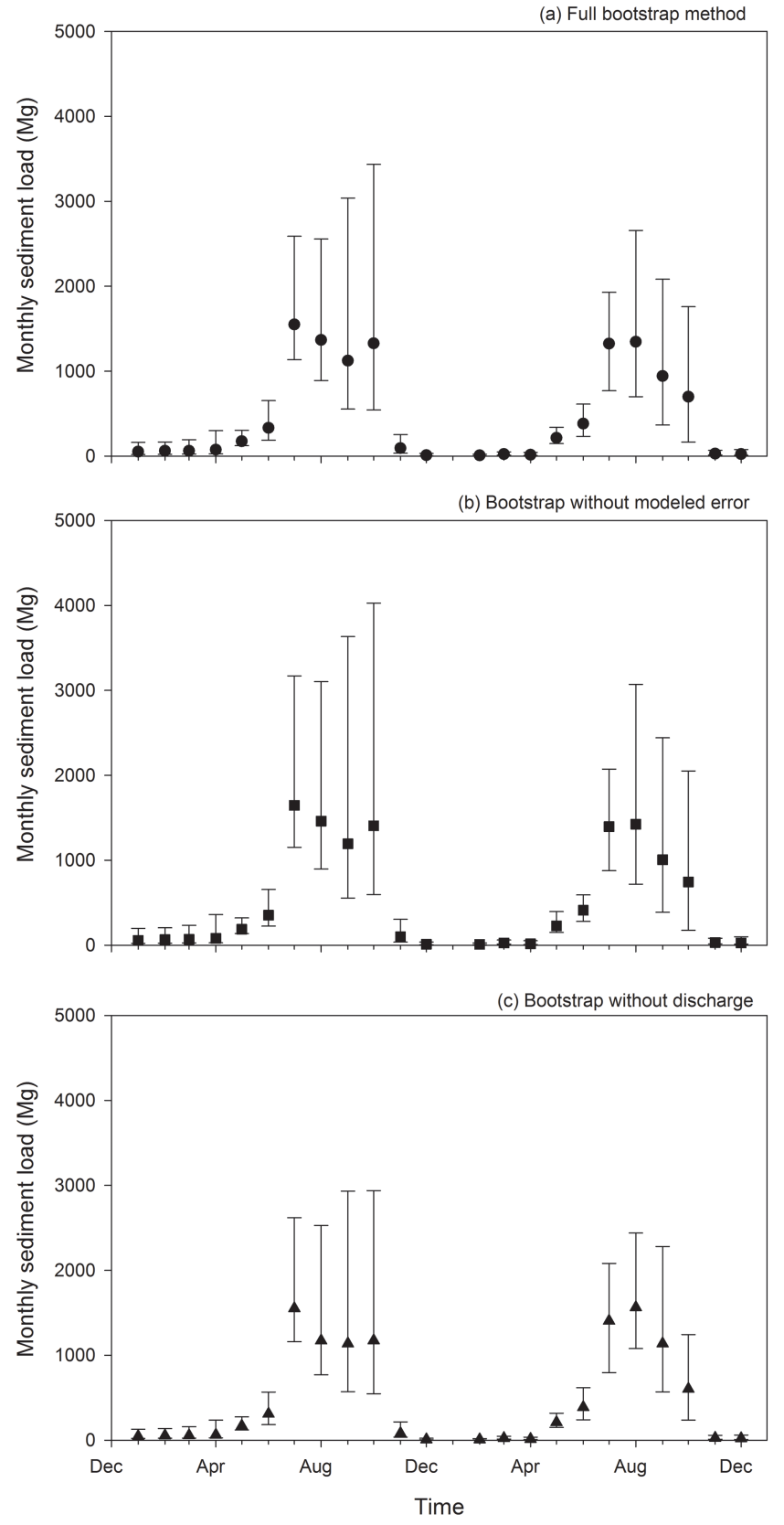

Figure 6. Monthly sediment load estimates (in $\mathrm{Mg}$ per year) for the 2 years of the study with $95 \%$ confidence interval limits for the three different bootstrap methods: (a) the full method shown in Fig. 1, (b) the method without modeled error (i.e., leaving out Step 3 in Fig. 1) and (c) the method without bootstrapping discharge (i.e., leaving out Step 1 in Fig. 1). In January 2011, discharge was zero; therefore, no sediment load was transported during this month.

2010 contained a smaller proportion of the samples (73 out of 228), and this could be a cause of the difference. For the monthly loads (Fig. 6), confidence intervals were widest during months with the highest sediment loads (July till October), when excess reservoir water gets exported via the river. 

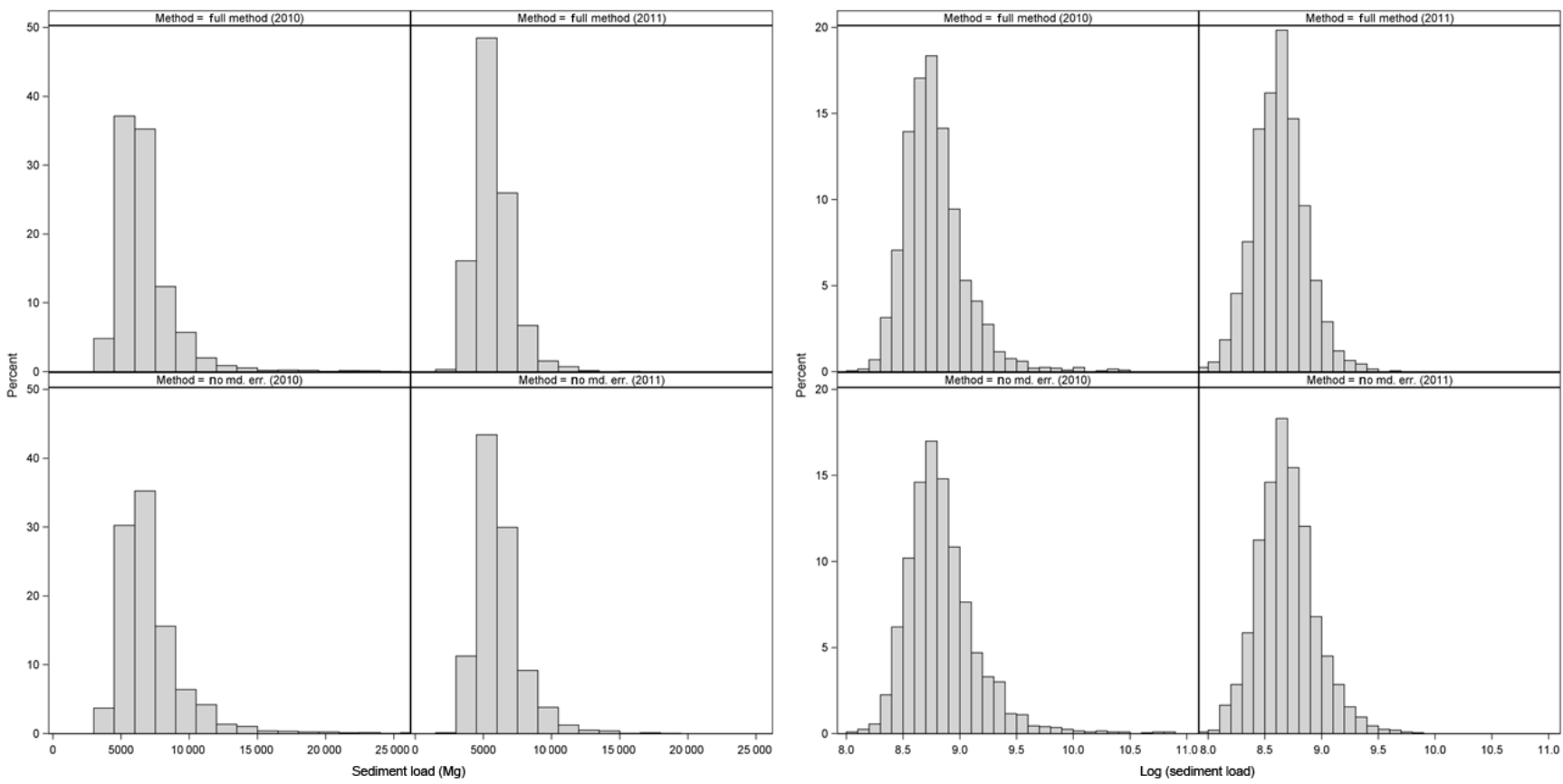

Figure 7. Histograms of bootstrap load estimates on the original scale (left) and the log scale (right) for 2 study years and for two bootstrap methods: the full method with modeling of the autocorrelated error ("full method", top), and without modeling of the error ("no md. err.", bottom).

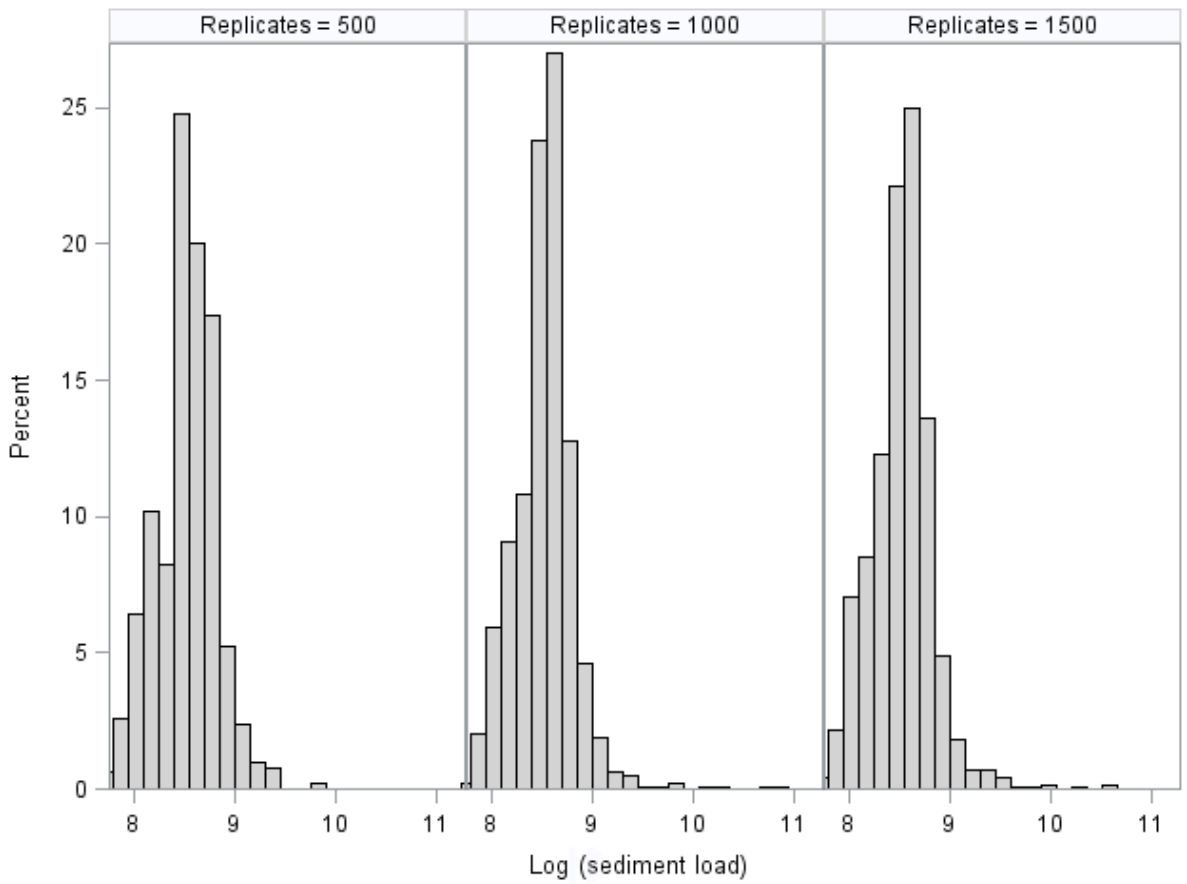

Figure 8. Effect of the number of bootstrap replicates (1500, 1000 and 500) on the smoothness of the resulting empirical distribution for the estimated annual sediment load in 2011.

The bootstrap method affected the width of the confidence interval as well. The monthly and annual intervals resulting from applying a back-transformation correction were consis- tently wider than those resulting from the bootstrap process that modeled the autocorrelated error: not modeling the error changed the interval (limits in $\mathrm{Mg}$ ) from $(4331,12267)$ 


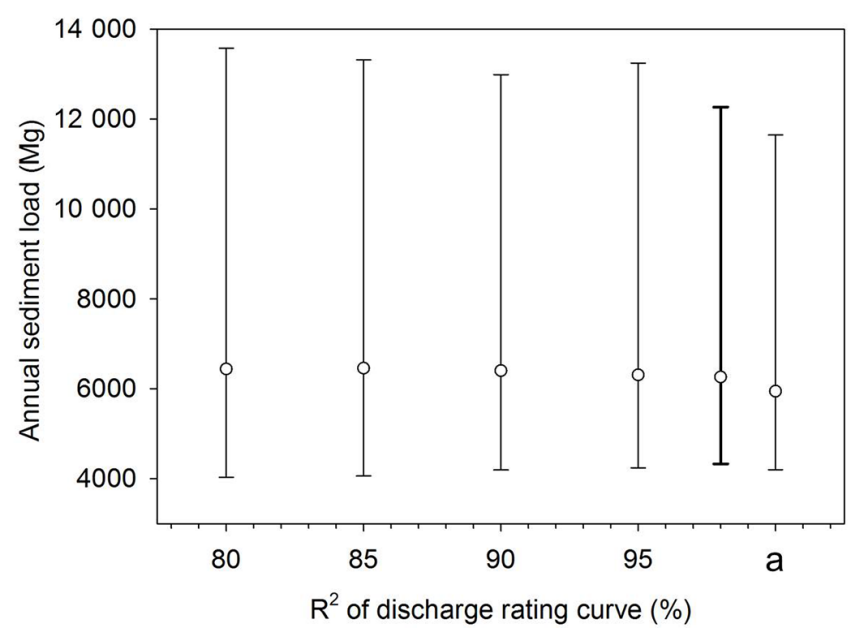

Figure 9. Change in the median and $95 \%$ confidence intervals for the sediment load estimate of 2010 (in $\mathrm{Mg}$ ) when decreasing the coefficient of determination of the discharge rating curve. The bold line indicates the CI width of the real (discharge, level) dataset. The letter "a" corresponds to not bootstrapping the (discharge, level) pairs.

to $(4372,14586)$ in 2010 and from $(3593,8975)$ to $(3713$, $10410)$ in 2011 - in both cases an increase in width of about $20 \%$. The change was due to an increase in the upper bound of the interval, while the lower limits remained very similar. These results show that performing the back-transformation correction is only a very rough method of adjusting the predicted concentrations on the original scale, as this approach does not take the serial correlation in the data into account. For the monthly load estimates, the largest differences in confidence interval width between the full method and the back-transformation without simulated error were in July and August 2010, the months with the highest estimated loads (Fig. 6).

\subsection{Hydrological drivers of uncertainty}

When, rather than applying the full bootstrap method, we did not bootstrap the discharge rating curve (meaning, we left out Step 1 of the process in Fig. 1), the width of the confidence interval decreased, as one less source of error is taken into account. In 2010, this changed the CI from (4203, 11649$)$ without accounting for uncertainty in the discharge rating curve to $(4331,12267)$ when accounting for this uncertainty on discharge; and from $(3521,8397)$ to $(3593,8975)$ in 2011 - including discharge therefore resulted in a respective increase in width of 6 and $9 \%$. Similarly, in the monthly load estimates, not bootstrapping the discharge resulted in confidence interval widths up to $37 \%$ smaller than those calculated with the full method (Fig. 6). Months with low flow showed equally compressed confidence intervals as months with high discharge, during which the reservoir spillover was feeding the river (July till October).
The accuracy in the $(Q, h)$ relationship in this particular dataset was very high, with an $R^{2}$ of 0.98 . As not all monitoring programs can establish accurate discharge rating curves, the $(Q, h)$ dataset was replaced with a simulated dataset with an increasingly lower coefficient of determination to test how this further affects the uncertainty in the load estimate (Fig. 9). While the width of the confidence interval keeps increasing with decreasing $R^{2}$, including the discharge also affects the confidence interval for a high $R^{2}$. In fact, changing from not bootstrapping the discharge $\left(R^{2}=100 \%\right.$ in Fig. 9) to bootstrapping the real discharge dataset, which has an $R^{2}$ of 0.98 , resulted in a $7 \%$ increase in width. On the other hand, the confidence intervals show little differences at an $R^{2}$ of 0.95 , where the width was $9003 \mathrm{Mg}$, and at 0.90 , when it reached up to $8795 \mathrm{Mg}$, equivalent to an $11 \%$ increase in width. At a coefficient of determination of 0.85 , the CI was $17 \%$ wider than the original CI, whereas at 0.80 the width increase reached $20 \%$. As can be seen from Fig. 9, the change in width was mainly due to an increase in the upper limit of the confidence interval. Hence, the lower limit decreased only slightly.

The bootstrap approach where the concentration prediction error was separated into an underlying latent autoregressive process generating the true concentrations, and an independently distributed measurement error corresponding to white noise in time series data, did not converge for 906 out of 2000 runs. Convergence problems are very common when trying to fit nugget models as these models tend to be difficult to fit. Particularly AR(1) type error structures are prone to these issues, as there is an inherent confounding between parameters of the independent white noise component and the autocorrelated component (Piepho et al., 2015). In a bootstrap setting where convergence was already an issue, adding such an effect was not feasible in the case of our dataset. For exploratory purposes, the nugget can be fitted to the original dataset without bootstrapping, in order to examine the contributions of the respective error components. Results of this exercise showed that indeed, the measurement error (0.67) was large compared to the latent process variance (0.09), the former being due to sensor error, both from the turbidity sensors and the pressure sensors for discharge, the manual grab sample process which may not accurately represent the mean concentration across the cross section, and laboratory error in determining the sediment concentrations. The error separation thus indicates that focusing on these factors could yield substantial improvement in the sediment rating curve.

\section{Discussion}

\subsection{Load estimates, data transformations and bias}

As was shown in Table 1, the annual sediment load estimates differ depending on the method selected. While it is encouraging that all estimates are within the $95 \%$ confidence in- 
terval limits, choosing a different method can lead to anything from an underestimation of $10 \%$ to an overestimation of $20 \%$ compared to the median of the full bootstrap process. Two issues play a role in these differences: the backtransformation of the sediment concentrations, and bias in the estimate of the annual load.

The effect of back-transforming the concentration predictions is visible when comparing the medians of the bootstrap estimates with and without modeled autocorrelated error. When the error was not modeled, the estimate itself increased by around $5 \%$ in both years, corresponding to an absolute increase of around $300 \mathrm{Mg}$ of sediment, and the CI became wider. Essentially, adding half the variance before backtransformation is a very rough way of estimating expected values of concentrations at observed time points - as shown by the larger CI - because it does not take the serial correlation in the data into account. If the naïve back-transformation would be applied, without any variance correction, the resulting estimates would be even lower than those where we add half the variance before back-transformation: around $4200 \mathrm{Mg}$ in 2010 and $3700 \mathrm{Mg}$ in 2011 , or an underestimation of approximately $2000 \mathrm{Mg}$.

While the latter is a relatively common approach to implement the back-transformation of constituent concentration predictions which are typically predicted on the log-scale, it may not be the most appropriate solution when the concentrations are used to calculate loads. The crucial issue with load calculations is that a load is a sum over time points, which is essentially the same as computing an arithmetic average, and for that we need to estimate the expected values for the individual time intervals. If the predicted value on the log-scale is simply back-transformed, we are estimating medians of the concentrations, and while this may be appropriate if one is only interested in the concentrations, these medians cannot be multiplied with discharge and summed up to accurately predict a load.

When the bootstrap process includes a simulated, autocorrelated error, the result of that process is not a mean or a median concentration, but rather a simulated realization of an observed process. When it is not desired to simulate the error in the bootstrap process, then applying a back-transformation correction is an alternative, but the confidence intervals should be expected to be wider, as adding on half the residual error variance before back-transformation ignores the serial correlation. An alternative back-transformation correction often used in the literature, Duan's smearing correction, similarly assumes independent and identically distributed errors and is therefore not suitable for datasets where serial correlation is present (Duan, 1983). Duan's is a non-parametric correction, in contrast to the two parametric approaches we used.

The back-transformation method of the concentration predictions, however, is not the only force at work: the direct estimate from the data and the bootstrap median without modeled error are quite far apart, even though they both use the same back-transformation correction. Statistics, unless they are very simple (for example a sample mean), will typically have some bias. Bootstrapping can in fact be used to identify and correct bias even when the true underlying distribution is unknown; therefore, in most cases the bootstrap estimate will typically be different, as it removes this bias (Efron and Tibshirani, 1993). There are alternative methods in the literature intended to remove the bias on load estimates (Ferguson, 1986), but as the correction will depend on the variance of the data, numerical corrections are not generally applicable. However, as one would need to bootstrap in any case in order to produce a CI of the load, taking the median of the bootstrap estimates is a straightforward way to obtain constituent load estimates.

The most common data transformations in load estimation are typically other variations of the Box-Cox power family, such as $1 / Y$, square root, cube root, and fourth root. Transformations in this family are usually required where the original data exhibit pronounced skewness and heteroscedasticity, which is generally the case in load studies. Therefore for all transformations in the Box-Cox family, naïve backtransformation of estimates would similarly result in biased estimates of means on the original scale, as was illustrated with the log-transformation in our dataset.

Regarding the data transformation, while the sediment concentration was log-normally distributed, the logtransformed load estimates were not normally distributed (Fig. 7, right panel). This non-log-normality of our loads does not affect the viability of the bootstrap approach, as regression-type methods do not require (log-) normality of the observed concentration or load data but rather normality of the residuals of the fitted linear regression models. It does, however, limit the applicability of methods that use the lognormality assumption of the load to estimate a variance for the load. This assumption was made, for example, by Wang et al. (2011) in using the delta method as an alternative way to assess uncertainty on annual sediment load estimates.

\subsection{Confidence interval width and model selection}

The results showed that the CIs are relatively wide and asymmetrical with a much larger uncertainty in the upper limit. And comparing the 2 years, when the estimated load was higher, the uncertainty in it was larger as well (i.e., in 2010). This is a trend consistent with other studies (Kuhnert et al., 2012; Rustomji and Wilkinson, 2008). Although it is difficult to compare uncertainty calculated with other methods in different catchments, our confidence intervals are on the same order of magnitude as the CIs in those two studies. For example, Kuhnert et al. (2012) calculated $80 \%$ confidence intervals on an annual load of $5232 \mathrm{Mg}(n=122)$, and the resulting limits were $(3512,7775)$. In comparison, $80 \%$ confidence limits for our dataset were $(4186,7403)$ for a load of $5543 \mathrm{Mg}$ in 2011. 
The factors governing the width of a confidence interval are essentially the sample size and the accuracy of the two rating curve estimates. The accuracy of the sediment rating curve in this study (Pearson's $r^{2}=0.56$ after cross validation) is reasonable for catchments with large heterogeneity in relief, land use, soil types and rainfall event characteristics. In more homogeneous settings, however, much more accurate sediment rating curves have been obtained, which can be expected to result in narrower confidence intervals on their resulting load estimates. Furthermore, if the sample size and the variation explained by the rating curves are large, but the confidence intervals are very wide, one possible cause is that the concentration prediction model was over-fitted, resulting in a very high apparent percentage of variance explained by the model but a poor predictive power when the model is interpolated to the whole time series. This can be shown by just adding additional predictor variables to our selected model. If we add the variables "water height in the reservoir", "discharge irrigated to the paddy fields" and "Julian day-of-year" (the last one both linear and quadratic) to the model, the percentage of variance explained increases from 58 to $71 \%$. When this extended model was used to estimate the annual sediment load, however, the confidence interval was inflated by 2 orders of magnitude, resulting in a width of $5564076 \mathrm{Mg}$.

These effects on the CI indicate that, indeed, overfitting is a concern even when interpolating within the time series. The risk of overfitting is particularly high with more complex models (Burnham and Anderson, 2002), as was demonstrated as well with the example above, and it is not uncommon in load estimations to fit models that are very flexible (e.g., spline functions, sigmoid functions) and/or have used a large number of predictor variables to a relatively small dataset. In such cases, bootstrap uncertainty assessment can be an additional tool both for model selection and for evaluating model fit. The change in percentage variance explained is less pronounced after cross validation, and ranges from 56 to $64 \%$, implying that the cross validation penalizes at least partially for any overfitting. Water quality models, however, are often not validated, and only the $R^{2}$ resulting from calibration is reported, leaving readers no means of assessing over-parameterization of the model. Studies with smaller datasets where more variables are included in the model should be particularly encouraged to report measures of uncertainty in load estimates. In the case of large datasets where a simple model such as linear regression with one or two predictor variables is used, the variability in the data explained by the model resulting from calibration only is less likely to deviate strongly from the result of a validation.

\subsection{Bootstrapping discharge and error propagation}

One would expect that, as the sediment rating curve has much more uncertainty than the discharge rating curve, excluding the latter would not affect the confidence intervals much, but for our dataset, this assumption did not hold: even with a discharge rating curve with high accuracy ( $R^{2}$ of 0.98$)$, its uncertainty had a considerable effect on the load estimate, which increased from $6389 \mathrm{Mg}$ when not bootstrapping the discharge to $6781 \mathrm{Mg}$ when bootstrapping the discharge.

This result underscores the importance of error propagation in uncertainty assessments. Even though the discharge rating curve has a high accuracy, an estimate of $Q$ is used as a predictor variable for concentration, and the concentration then gets multiplied by the estimate of $Q$, and so the effect is not as small as one would expect based on the $R^{2}$ of both rating curves. It is possible that the sample size of the discharge rating curve, which is relatively small $(n=15)$, plays a role here, as a bootstrap iteration that does not contain the largest discharge values would result in a wider confidence interval for the estimated load. Krueger et al. (2009) similarly found discharge to contribute to uncertainty of sediment transfer. If we assume that most discharge rating curves have around $95 \%$ of the explained variance, this could imply that most measures of uncertainty in the literature are too conservative by about $10 \%$ in terms of the width of the CI and that this increase would be mostly on the upper limit of the interval - implying that minimum impact estimates would not be affected much, but that the literature to date would underestimate worst case scenarios of sediment yield, nutrient loss or erosion by about $10 \%$.

\section{Conclusions}

The approach developed in this paper provides a means to assess uncertainty in any type of constituent load, which was calculated from continuous constituent concentration and discharge predictions estimated with regression-type methods. Compared to ordinary least squares regression methods to obtain load estimates, bootstrap estimates resulted in biascorrected estimates that can take serial correlation into account when present as well as provide a measure of uncertainty in the load estimate.

The results show that, even when the uncertainty of the discharge rating curve is small, it is important to take into account that the errors propagate by using discharge both as a predictor variable for constituent concentration and in the instantaneous load equation. Application of the method in different watersheds, at different spatial and temporal scales, could elucidate whether discharge is an important driver of uncertainty in those settings as well.

The confidence intervals resulting from our proposed method showed that the uncertainty in the loads is quite large and is mostly on the upper limit of the estimate, as the intervals were strongly right-skewed. This asymmetry implies that, wherever load estimates are used to assess environmental impact, without reporting an uncertainty assessment, the maximum impact could be severely underestimated. 
Additionally, the bootstrap process demonstrated that load estimates are biased downwards if calculated directly from data with increasing variance that has been transformed. While some alternative bias corrections are available, these are not consistently used, and this is another factor contributing to the underestimation of constituent loads thus far reported in the literature. Taking the median of the bootstrap estimates is an easy and generally applicable way to obtain unbiased estimates.

Reporting uncertainty is especially important when water quality models are complex. There has been a great increase in the use of more complex predictive methods for water quality, for example, the use of artificial neural networks, random forests or generalized additive models (Berk, 2008). The advent of these methods makes the consistent reporting of measures of uncertainty even more essential: the more complex a model is, the more prone it is to overfitting (Burnham and Anderson, 2002), as was demonstrated by the inflated confidence intervals when adding predictor variables to the sediment concentration model. Some measure of uncertainty should systematically be shown for any load estimate, and the method developed in this paper provides a flexible framework to do so.

\section{Data availability}

The source code for the bootstrap analysis with the SAS software that was used for the load estimates and corresponding confidence intervals is freely available at https://www. uni-hohenheim.de/bioinformatik/beratung/index.htm (Slaets et al., 2016) together with the necessary input files for testing. The full dataset is available from the authors upon request (hanna.slaets@gmail.com).

The SAS code used to simulate a dataset with a fixed realized $R^{2}$ can be found in the Supplement. 


\section{Appendix A}

In order to obtain $(\log (Q), \log (h))$ pairs with a certain $R^{2}$ (e.g., 0.95), we started by simulating a dataset of the same number of observations of the original dataset. We thus obtained pairs of $y_{i}=\log \left(Q_{i}\right)$ and $x_{i}=\log \left(h_{i}\right)$ using the original discharge rating curve, which is a regression model $y_{i}=\eta_{i}+e_{i}$, where $\eta_{i}=\alpha+\beta x_{i}$ and the errors were randomly drawn from a normal distribution which can have any variance, as the errors will be rescaled later.

Next, we computed the total sum of squares, $\mathrm{SS}_{y}$, and the residual sum of squares, $\mathrm{SS}_{e}$, for this simulated dataset. We subsequently replaced the simulated errors $e_{i}$ by re-scaled errors $e_{i}^{*}=c e_{i}$ and used these to compute re-scaled simulated data $y_{i}^{*}=\eta_{i}+e_{i}^{*}$. A scaling constant $c$ was chosen in such a way that the desired coefficient of determination results:

$R^{2}=1-\mathrm{SS}_{e} / \mathrm{SS}_{y}$ and $R^{2 *}=1-\mathrm{SS}_{e}^{*} / \mathrm{SS}_{y}^{*}$.

The residual error sum of squares is a quadratic form of errors only. It follows that

$\mathrm{SS}_{e}^{*}=c^{2} \mathrm{SS}_{e}$,

$$
\begin{aligned}
\mathrm{SS}_{y}^{*} & =\sum_{i=1}^{n}\left(y_{i}^{*}-\bar{y}_{.}^{*}\right)^{2}=\sum_{i=1}^{n}\left(\eta_{i}+c e_{i}-\bar{\eta} .-c \bar{e} .\right)^{2} \\
& =\sum_{i=1}^{n}\left[\left(\eta_{i}-\bar{\eta} .\right)+c\left(e_{i}-\bar{e}_{.}\right)\right]^{2} \\
& =\sum_{i=1}^{n}\left(\eta_{i}-\bar{\eta} .\right)^{2}+2 c \sum_{i=1}^{n}\left(\eta_{i}-\bar{\eta} .\right)\left(e_{i}-\bar{e}_{.}\right) \\
& +c^{2} \sum_{i=1}^{n}\left(e_{i}-\bar{e}_{.}\right)^{2}=z_{1}+z_{2} c+z_{3} c^{2}
\end{aligned}
$$

where $z_{1}, z_{2}$ and $z_{3}$ are computable constants for given simulated $\left(\eta_{i}, e_{i}\right)$.

$$
\begin{aligned}
R^{2 *} & =1-\mathrm{SS}_{e}^{*} / \mathrm{SS}_{y}^{*} \Leftrightarrow \\
0 & =\mathrm{SS}_{e}^{*} / \mathrm{SS}_{y}^{*}-1+R^{2 *} \Leftrightarrow \\
0 & =\mathrm{SS}_{e}^{*}+\left(R^{2 *}-1\right) \mathrm{SS}_{y}^{*} \\
& =c^{2} \mathrm{SS}_{e}+\left(R^{2 *}-1\right)\left(z_{1}+z_{2} c+z_{3} c^{2}\right) \\
& =A c^{2}+B c+C
\end{aligned}
$$

This is a quadratic equation in $c$, which can be solved for $c$ by standard procedures. There are two distinct solutions, but they result in errors that only differ in the sign, and so either solution can be chosen.

SAS code to perform this simulation can be found in the Supplement of this paper. 


\section{The Supplement related to this article is available online at doi:10.5194/hess-21-571-2017-supplement.}

Acknowledgements. The fieldwork data in this study were collected within the framework of the Uplands Program collaborative research center, a DFG-funded project in collaboration with Tran Duc Vien at the Hanoi University of Agriculture. The authors gratefully acknowledge the work of field assistants Do Thi Hoan and Nguyen Duy Nhiem, and the laboratory analyses were done at the Central Water and Soil Lab of the Hanoi University of Agriculture, under supervision of Nguyen Huu Thanh by Dang Thi Thanh Hue and Phan Linh. Finally we thank the two reviewers for their thoughtful insights on this paper.

Edited by: S. Archfield

Reviewed by: T. Kumke and one anonymous referee

\section{References}

ASTM: Standard D3977-97, Standard test methods for determining sediment concentration in water samples, ASTM International, West Conshohocken, PA, 7 pp., 2013.

Berk, R. A.: Statistical learning from a regression perspective, Springer, New York, ISBN-10: 0387775013, 347 pp., 2008.

Burnham, K. P. and Anderson, D. R.: Model selection and multimodel inference: a practical information-theoretic approach, Springer, New York, ISBN-10: 0387953647, 454 pp., 2002.

Clemens, G., Fiedler, S., Cong, N. D., Van Dung, N., Schuler, U., and Stahr, K.: Soil fertility affected by land use history, relief position, and parent material under a tropical climate in NWVietnam, Catena, 81, 87-96, 2010.

Duan, N.: Smearing estimate: a nonparametric retransformation method, J. Am. Stat. Assoc., 78, 605-610, 1983.

Ebtehaj, M., Moradkhani, H., and Gupta, H. V.: Improving robustness of hydrologic parameter estimation by the use of moving block bootstrap resampling, Water Resour. Res., 46, W07515, doi:10.1029/2009WR007981, 2010.

Efron, B. and Tibshirani, R. J.: An introduction to the bootstrap, Chapman \& Hall/CRC, Boca Raton, ISBN-13: 9780412042317 , 436 pp., 1993.

Ferguson, R. I.: River loads underestimated by rating curves, Water Resour. Res., 22, 74-76, 1986.

Freeman, J. and Modarres, R.: Inverse Box-Cox: the power-normal distribution, Stat. Probabil. Lett., 76, 764-772, 2006.

Gao, P.: Understanding watershed suspended sediment transport, Prog. Phys. Geog., 32, 243-263, 2008.

Hamilton, A. S. and Moore, R. D.: Quantifying uncertainty in streamflow records, Can. Water Resour. J., 37, 3-21, 2012.

Harmel, R. D., Smith, D. R., King, K. W., and Slade, R. M.: Estimating storm discharge and water quality data uncertainty: A software tool for monitoring and modeling applications, Environ. Modell. Softw., 24, 832-842, 2009.

Herschy, R. W.: Streamflow measurement, CRC Press, Boca Raton, ISBN-13: 978-0-415-41342-8, 507 pp., 1995.
Hirsch, R. M., Archfield, S. A., and De Cicco, L. A.: A bootstrap method for estimating uncertainty of water quality trends, Environ. Modell. Softw., 73, 148-166, 2015.

Horowitz, A. J.: Determining annual suspended sediment and sediment-associated trace element and nutrient fluxes, Sci. Total Environ., 400, 315-343, 2008.

Krueger, T., Quinton, J. N., Freer, J., Macleod, C. J., Bilotta, G. S., Brazier, R. E., Butler, P., and Haygarth, P. M.: Uncertainties in data and models to describe event dynamics of agricultural sediment and phosphorus transfer, J. Environ. Qual., 38, 11371148, 2009.

Kuhnert, P. M., Henderson, B. L., Lewis, S. E., Bainbridge, Z. T., Wilkinson, S. N., and Brodie, J. E.: Quantifying total suspended sediment export from the Burdekin River catchment using the loads regression estimator tool, Water Resour. Res., 48, W04533, doi:10.1029/2011WR011080, 2012.

Kulasova, A., Smith, P. J., Beven, K. J., Blazkova, S. D., and Hlavacek, J.: A method of computing uncertain nitrogen and phosphorus loads in a small stream from an agricultural catchment using continuous monitoring data, J. Hydrol., 458-459, 18, 2012.

Lahiri, S. N.: Resampling methods for dependent data, Springer, New York, ISBN-13: 978-1-4419-1848-2, 2003.

Lessels, J. S. and Bishop, T. F. A.: Estimating water quality using linear mixed models with stream discharge and turbidity, J. Hydrol., 498, 13-22, 2013.

Mailhot, A., Rousseau, A. N., Talbot, G., Gagnon, P., and Quilbé, R.: A framework to estimate sediment loads using distributions with covariates: Beaurivage River watershed (Québec, Canada), Hydrol. Process., 22, 4971-4985, 2008.

McMillan, H., Krueger, T., and Freer, J.: Benchmarking observational uncertainties for hydrology: rainfall, river discharge and water quality, Hydrol. Process., 26, 4078-4111, 2012.

Moatar, F. and Meybeck, M.: Compared performances of different algorithms for estimating annual nutrient loads discharged by the eutrophic River Loire, Hydrol. Process., 19, 429-444, 2005.

Nearing, M. A.: Why soil erosion models over-predict small soil losses and under-predict large soil losses, Catena, 32, 15-22, 1998.

Némery, J., Mano, V., Coynel, A., Etcheber, H., Moatar, F., Meybeck, M., Belleudy, P., and Poirel, A.: Carbon and suspended sediment transport in an impounded alpine river (Isère, France), Hydrol. Process., 27, 2498-2508, 2013.

Pagendam, D. E., Kuhnert, P. M., Leeds, W. B., Wikle, C. K., Bartley, R., and Peterson, E. E.: Assimilating catchment processes with monitoring data to estimate sediment loads to the great barrier reef, Environmetrics, 25, 214-229, 2014.

Patterson, H. D. and Thompson, R.: Recovery of inter-block information when block sizes are unequal, Biometrika, 58, 545-554, 1971.

Piepho, H. P.: Data transformation in statistical analysis of field trials with changing treatment variance, Agron. J., 101, 865-869, doi:10.2134/agronj2008.0226x, 2009.

Piepho, H. P., Möhring, J., Pflugfelder, M., Hermann, W., and Williams, E. R.: Problems in parameter estimation for power and AR(1) models of spatial correlation in designed field experiments, Communications in Biometry and Crop Science, 10, 3 16,2015 . 
Rustomji, P. and Wilkinson, S. N.: Applying bootstrap resampling to quantify uncertainty in fluvial suspended sediment loads estimated using rating curves, Water Resour. Res., 44, W09435, doi:10.1029/2007WR006088, 2008.

Sauer, V. B. and Meyer, R. W.: Determination of error in individual discharge measurements, US Department of the Interior, US Geological Survey, Washington, DC, 21 pp., 1992.

Schmitter, P., Fröhlich, H. L., Dercon, G., Hilger, T., Huu Thanh, N., Lam, N. T., Vien, T. D., and Cadisch, G.: Redistribution of carbon and nitrogen through irrigation in intensively cultivated tropical mountainous watersheds, Biogeochemistry, 109, 133-150, 2012.

Selle, B. and Hannah, M.: A bootstrap approach to assess parameter uncertainty in simple catchment models, Environ. Modell. Softw., 25, 919-926, 2010.

Slaets, J. I. F., Schmitter, P., Hilger, T., Lamers, M., Piepho, H. P., Vien, T. D., and Cadisch, G.: A turbidity-based method to continuously monitor sediment, carbon and nitrogen flows in mountainous watersheds, J. Hydrol., 513, 45-57, 2014.

Slaets, J. I. F., Piepho, H. P., Schmitter, P., Hilger, T., and Cadisch, G.: Quantifying uncertainty on sediment loads using bootstrap confidence intervals, available at: https://www. uni-hohenheim.de/bioinformatik/beratung/index.htm (last access: 21 January 2017), 2016.

Smith, C. and Croke, B.: Sources of uncertainty in estimating suspended sediment load, IAHS-AISH Publication, 292, 136-143, 2005.
Tomkins, K. M.: Uncertainty in streamflow rating curves: Methods, controls, and consequences, Hydrol. Processes, 28, 464481, 2014.

Verbeke, G. and Molenberghs, G.: Linear mixed models for longitudinal data, Springer, New York, ISBN-10: 1441903003, 484 pp., 2009.

Vigiak, O. and Bende-Michl, U.: Estimating bootstrap and Bayesian prediction intervals for constituent load rating curves, Water Resour. Res., 49, 8565-8578, 2013.

Walling, D. E.: Limitations of the rating curve technique for estimating suspended sediment loads, with particular reference to British rivers, IAHS-AISH Publication, 122, 34-118, 1977.

Walling, D. E. and Webb, B. W.: The reliability of rating curve estimates of suspended sediment yield: Some further comments, in: Variability in stream erosion and sediment transport, edited by: Olive, L. J., Loughran, R. J., and Kesby, J. A., IAHS Publ., 269-279, 1988.

Walling, D. E. and Webb, B. W.: Erosion and sediment yield: A global overview, IAHS-AISH Publication, 236, 3-19, 1996.

Wang, Y. G., Kuhnert, P., and Henderson, B.: Load estimation with uncertainties from opportunistic sampling data - A semiparametric approach, J. Hydrol., 396, 148-157, 2011. 